\title{
Mathematical Modeling and Simulation of Antibubble Dynamics
}

\author{
Junxiang Yang ${ }^{1}$, Yibao $\mathrm{Li}^{2}$, Darae Jeong ${ }^{3}$ and Junseok Kim ${ }^{1, *}$ \\ ${ }^{1}$ Department of Mathematics, Korea University, Seoul 02841, Republic of \\ Korea \\ ${ }^{2}$ School of Mathematics and Statistics, Xi'an Jiaotong University, Xi'an 710049, \\ China \\ ${ }^{3}$ Department of Mathematics, Kangwon National University, Gangwon-do \\ 24341, Republic of Korea
}

Received 10 June 2019; Accepted (in revised version) 30 August 2019

\begin{abstract}
In this study, we propose a mathematical model and perform numerical simulations for the antibubble dynamics. An antibubble is a droplet of liquid surrounded by a thin film of a lighter liquid, which is also in a heavier surrounding fluid. The model is based on a phase-field method using a conservative Allen-Cahn equation with a space-time dependent Lagrange multiplier and a modified Navier-Stokes equation. In this model, the inner fluid, middle fluid and outer fluid locate in specific diffusive layer regions according to specific phase filed (order parameter) values. If we represent the antibubble with conventional binary or ternary phase-field models, then it is difficult to have stable thin film. However, the proposed approach can prevent nonphysical breakup of fluid film during the simulation. Various numerical tests are performed to verify the efficiency of the proposed model.
\end{abstract}

AMS subject classifications: 35Q35, 76T10, 81T80

Key words: Antibubble, conservative Allen-Cahn equation, Navier-Stokes equation.

\section{Introduction}

In this paper, we propose a mathematical model for the antibubble dynamics and perform several numerical simulations. An antibubble is a droplet of liquid surrounded by a thin film of a lighter liquid, which is also in a heavier surrounding fluid, see Fig. 1 for the antibubble formation.

The terminology of antibubble first occurred in [2] and the antibubble can be generated in many fluids, such as diesel oil [3], soapy water, Belgian beer [4], etc. Unlike a single bubble system, the antibubble system consists of three fluid components: inner core fluid (fluid 1), middle fluid film (fluid 2) and outer ambient fluid (fluid 3). Usually,

\footnotetext{
${ }^{*}$ Corresponding author. Email address: cfdkim@korea.ac.kr (J. Kim)
} 


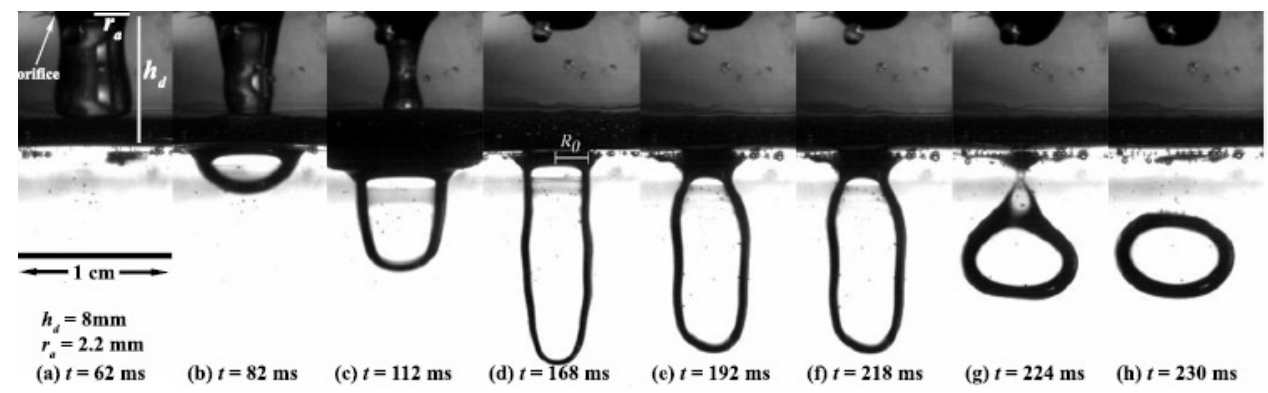

Figure 1: Formation of an antibubble. Reprinted from [1], with permission from IOP Publishing LTD.

the inner fluid and the outer fluid are the same. Because of the special fluid structure, the form of antibubble can be applied in some scientific, industrial and medical fields, including mass transfer of air [5], fluid transportation in a magnetic field [6], emulsion formation [7]. Furthermore, if the inner fluid is different from the outer fluid, then the antibubble structure can be extended for liquid drag delivery [8].

Recently, some researchers have conducted many theoretical and experimental studies on the antibubble. Scheid et al. [9] proposed a dynamic model for explaining the effect of surface shear viscosity on the drainage of air film. Sob'yanin [10] proposed a theory of antibubble collapse to account for the motion of air rim which can not be well explained by potential flow. Zou et al. [11] investigated the collapse of an antibubble, they found that the small bubbles are generated along the rim of air film during the collapse process. Kim and Stone [1] experimentally studied the dynamics of antibubble and provided the optimal condition for antibubble formation. Bai et al. [12] proposed a new experimental method for antibubble formation. However, to the best knowledge of authors, there are few numerical investigations of antibubble. Kim [13] performed a simulation of antibubble raising by using ternary Cahn-Hilliard-Navier-Stokes system, while the middle fluid film is set thick and the stability can not be satisfied in a long time.

In this paper, we propose a mathematical model for antibubble simulation using phase-filed method. Phase-field method is a popular issue in multiphase flow field and large amounts of researches have been studied by some scholars [14-18]. The proposed model is based on the conservative Allen-Cahn equation [19], which can avoid mass spreading to the bulk phase. In this model, the inner fluid locates in the region where the order parameter $\phi \geq 0$, the middle fluid film occupies the region where $-0.9<\phi<0$ and the rest of the region is filled with the outer ambient fluid. This approach can naturally keep a stable thin fluid film.

The remaining parts of this paper are organized as follows. In Section 2, we give the mathematical model. The numerical solution of the proposed model is presented in Section 3. To validate the new proposed model, various numerical tests are performed in Section 4. In Section 5, conclusions are drawn. 


\section{Mathematical model}

\subsection{Governing equation}

We consider the following system modeling viscous, immiscible, incompressible, two-phase fluid flow with surface tension force:

$$
\begin{aligned}
& \rho(\phi)\left(\mathbf{u}_{t}+\mathbf{u} \cdot \nabla \mathbf{u}\right)=-\nabla p+\frac{1}{R e} \nabla \cdot\left[\eta(\phi)\left(\nabla \mathbf{u}+\nabla \mathbf{u}^{T}\right)\right]+2 \mathbf{S F}(\phi)+\frac{\rho(\phi)}{F r} \mathbf{g}, \\
& \nabla \cdot \mathbf{u}=0, \\
& \phi_{t}+\nabla \cdot(\phi \mathbf{u})=\frac{1}{P e}\left(-F^{\prime}(\phi)+\epsilon^{2} \Delta \phi\right)+\beta(t) \sqrt{F(\phi)},
\end{aligned}
$$

where $\mathbf{u}$ is the fluid velocity, $p$ is the pressure, $\rho(\phi)$ and $\eta(\phi)$ are the variable density and viscosity, respectively. $F(\phi)=0.25\left(\phi^{2}-1\right)^{2}, \epsilon$ is a positive constant and $\beta(t)$ is a nonconstant Lagrange multiplier which enforces the mass conservation. In an antibubble system, the surface tension coefficients $\sigma_{12}$ and $\sigma_{23}$ play a joint effect, where $\sigma_{12}$ and $\sigma_{23}$ are the surface tension coefficients on the interface between fluid 1 and fluid 2 and on the interface between fluid 2 and fluid 3, respectively, see schematic illustration in Fig. 2. For simplicity, we let fluid 1 and fluid 3 be the same (i.e., $\sigma_{12}=\sigma_{23}$ ), therefore, we take the double effect of surface tension in Eq. (2.1a). The formulas of density and viscosity will be introduced in Section 2.2. $\mathbf{g}=(0,-1)$ is the gravity, $\mathbf{S F}(\phi)$ is the surface tension of the single layer interface. The interfacial force $\mathbf{S F}(\phi)$ is given as [20]:

$$
\mathbf{S F}(\phi)=-\frac{3 \sqrt{2} \epsilon}{4 W e} \nabla \cdot\left(\frac{\nabla \phi}{|\nabla \phi|}\right)|\nabla \phi| \nabla \phi
$$

The dimensionless parameters are the Reynolds number, $R e=\rho_{c} U_{c} L_{c} / \eta_{c}$, the Weber number, $W e=\rho_{c} L_{c} U_{c}^{2} / \sigma$, the Froude number, $F r=U_{c}^{2} /\left(g L_{c}\right)$ and the Peclet number, $P e=U_{c} L_{c} /\left(M \mu_{c}\right)$, where $L_{c}, U_{c}, \rho_{c}, \eta_{c}, \sigma, M$ and $\mu_{c}$ are the characteristic length, velocity, density, viscosity, surface tension coefficient, constant mobility and chemical potential, respectively. Unless otherwise specified, we define those characteristic variables as $L_{c}=l, U_{c}=\sqrt{g l}, \rho_{c}=\rho_{1}, \eta_{c}=\eta_{1}$, where $l$ is chosen as the half of $x$-directional length, $\rho_{1}$ and $\eta_{1}$ are the density and viscosity of fluid 1, respectively. More details about the dimensionless process, please see [21].

\subsection{Density and viscosity formulas}

In this paper, we use the conservative Allen-Cahn-Navier-Stokes system to simulate the antibubble raising. Unless specific needs, we set the fluid 1 is surrounded by the fluid 2 in the ambient fluid 3. A diffusive region where $-0.9<\phi<0$ is used to represent the fluid 2, fluid 1 locates in the regions where $\phi \geq 0$ and the rest of the region is fluid 3, see Fig. 2. The density $\rho(\phi)$ and viscosity $\eta(\phi)$ are represented as the 


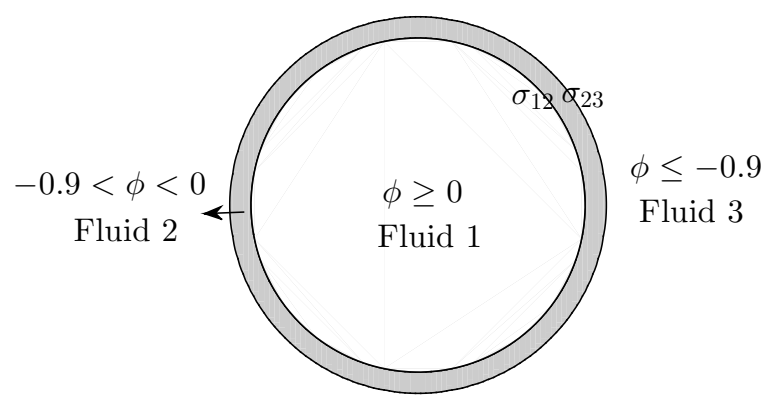

Figure 2: Schematic illustration of antibubble.

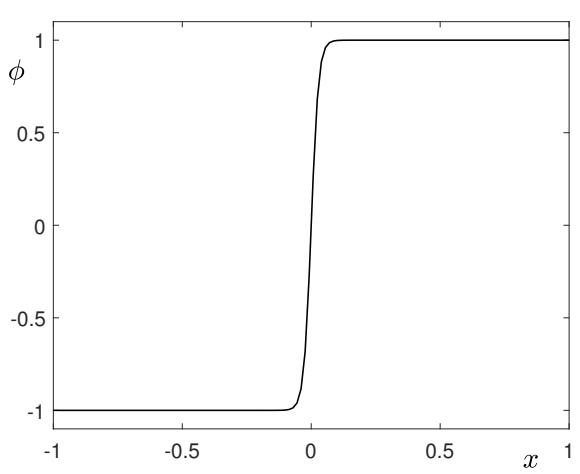

(a)

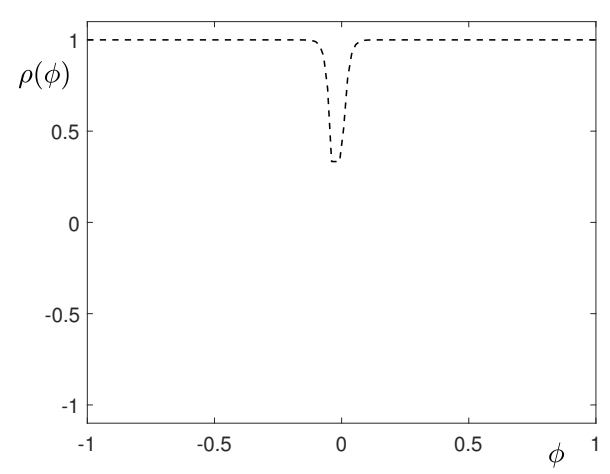

(b)

Figure 3: Schematic illustrations of (a) order parameter $\phi$ and (b) density $\rho(\phi)$ in one-dimensional space.

step functions of order parameter $\phi$. The density is defined as

$$
\rho(\phi)= \begin{cases}{\left[10\left(\rho_{2}-\rho_{3}\right)(\phi+1)+\rho_{3}\right] / \rho_{1},} & \text { if } \phi \leq-0.9, \\ \rho_{2} / \rho_{1}, & \text { if }-0.9<\phi<0, \\ {\left[\left(\rho_{1}-\rho_{2}\right) \phi+\rho_{2}\right] / \rho_{1},} & \text { otherwise, }\end{cases}
$$

where $\rho_{1}, \rho_{2}$ and $\rho_{3}$ are the densities of fluid 1 (inner), fluid 2 (middle) and fluid 3 (outer), respectively. In particular, the densities of the inner and outer fluids are the same, therefore, $\rho_{1}=\rho_{3}$. The variable viscosity is defined in the same manner. The illustrations of order parameter $\phi$ and density $\rho(\phi)$ in one-dimensional space are shown in Figs. 3(a) and (b), respectively, where the thick solid line represents the order parameter $\phi$ and the dash-dotted line represents the density. Note that the extension of the definition of the order parameter was used in modeling capillaries, membrane and extracellular matrix [22].

\section{Numerical solutions}

The proposed numerical method is composed of two parts: A projection method for the modified Navier-Stokes equation [23] and a hybrid method for the conservative 
Allen-Cahn equation with a space-time dependent Lagrange multiplier [24]. Let $\Omega=$ $(a, b) \times(c, d)$ be a computational domain and $h=(b-a) / N_{x}=(d-c) / N_{y}$ be a grid size, where $N_{x}$ and $N_{y}$ are even integers. For $i=1, \cdots, N_{x}$ and $j=1, \cdots, N_{y}$, let $\left(x_{i}, y_{j}\right)=$ $(a+(i-0.5) h, c+(j-0.5) h)$ be the cell centers. Let $\phi_{i j}^{n}$ and $p_{i j}^{n}$ be approximations of $\phi\left(x_{i}, y_{j}, n \Delta t\right)$ and $p\left(x_{i}, y_{j}, n \Delta t\right)$, respectively, where $\Delta t$ is the temporal step size. Velocities $u_{i+\frac{1}{2}, j}$ and $v_{i, j+\frac{1}{2}}$ are defined at cell edges $\left(x_{i+\frac{1}{2}}, y_{j}\right)=(a+i h, c+(j-0.5) h)$ and $\left(x_{i}, y_{j+\frac{1}{2}}\right)=(a+(i-0.5) h, c+j h)$, respectively.

Given $\mathbf{u}^{n}$, and $\phi^{n}$, we want to find $\mathbf{u}^{n+1}, \phi^{n+1}$ and $p^{n+1}$ which solve Eqs. (2.1a)(2.1c). Initialize $\mathbf{u}^{0}$ to be the divergence-free velocity field and $\phi^{0}$ to be the locally equilibrated concentration profile.

Step 1. Solve the intermediate velocity field $\tilde{\mathbf{u}}$ :

$$
\frac{\tilde{\mathbf{u}}-\mathbf{u}^{n}}{\Delta t}=\frac{1}{\rho^{n} R e} \nabla_{d} \cdot\left[\eta^{n}\left(\nabla_{d} \mathbf{u}^{n}+\left(\nabla_{d} \mathbf{u}^{n}\right)^{T}\right)\right]+\frac{2}{\rho^{n}} \mathbf{S F}^{n}+\frac{1}{F r} \mathbf{g}-\left(\mathbf{u} \cdot \nabla_{d} \mathbf{u}\right)^{n},
$$

where discretizations of the surface tension force $\mathbf{S F}^{n}$ and the advection term can be found in $[20,21]$. Next, we solve Eqs. (3.1a) and (3.1b) for the pressure field at $(n+1)$ time level.

$$
\begin{aligned}
& \frac{\mathbf{u}^{n+1}-\tilde{\mathbf{u}}}{\Delta t}=-\frac{1}{\rho^{n}} \nabla_{d} p^{n+1}, \\
& \nabla_{d} \cdot \mathbf{u}^{n+1}=0 .
\end{aligned}
$$

After taking the discrete divergence operator to Eq. (3.1a), we have the following Poisson equation

$$
\nabla_{d} \cdot\left(\frac{1}{\rho^{n}} \nabla_{d} p^{n+1}\right)=\frac{1}{\Delta t} \nabla_{d} \cdot \tilde{\mathbf{u}},
$$

where we used Eq. (3.1b). The resulting linear system of Eq. (3.2) is solved using a multigrid method [25].

Step 2. Update the phase-field from $\phi^{n}$ to $\phi^{n+1}$ : To solve Eq. (2.1c), we use an operator splitting method [24]:

$$
\begin{aligned}
\frac{\partial \phi}{\partial t} & =\frac{\epsilon^{2}}{P e} \Delta \phi-\nabla \cdot(\phi \mathbf{u}), \\
\frac{\partial \phi}{\partial t} & =-\frac{F^{\prime}(\phi)}{P e} \\
\frac{\partial \phi}{\partial t} & =\beta(t) \sqrt{F(\phi)}
\end{aligned}
$$

First, we solve Eq. (3.3a) by the following semi-implicit scheme with the multigrid method [25]:

$$
\frac{\phi_{i j}^{*}-\phi_{i j}^{n}}{\Delta t}=\frac{\epsilon^{2}}{P e} \Delta_{d} \phi_{i j}^{*}-\nabla_{d} \cdot(\phi \mathbf{u})_{i j}^{n}
$$


Next, we solve Eq. (3.3b) analytically using the method of separation of variables

$$
\phi_{i j}^{* *}=\frac{\phi_{i j}^{*}}{\sqrt{e^{-\frac{2 \Delta t}{P e}}+\left(\phi_{i j}^{*}\right)^{2}\left(1-e^{-\frac{2 \Delta t}{P e}}\right)}} .
$$

Eq. (3.3c) is discretized as

$$
\frac{\phi_{i j}^{n+1}-\phi_{i j}^{* *}}{\Delta t}=\beta^{* *} \sqrt{F\left(\phi_{i j}^{* *}\right)}
$$

Using Eq. (3.6) and the mass conservation, we have

$$
\sum_{i=1}^{N_{x}} \sum_{j=1}^{N_{y}} \phi_{i j}^{0}=\sum_{i=1}^{N_{x}} \sum_{j=1}^{N_{y}} \phi_{i j}^{n+1}=\sum_{i=1}^{N_{x}} \sum_{j=1}^{N_{y}}\left(\phi_{i j}^{* *}+\Delta t \beta^{* *} \sqrt{F\left(\phi_{i j}^{* *}\right)}\right) .
$$

Thus,

$$
\beta^{* *}=\frac{1}{\Delta t} \sum_{i=1}^{N_{x}} \sum_{j=1}^{N_{y}}\left(\phi_{i j}^{0}-\phi_{i j}^{* *}\right) / \sum_{i=1}^{N_{x}} \sum_{j=1}^{N_{y}} \sqrt{F\left(\phi_{i j}^{* *}\right)}
$$

Finally, we get $\phi^{n+1}$ from Eq. (3.6), i.e., $\phi_{i j}^{n+1}=\phi_{i j}^{* *}+\Delta t \beta^{* *} \sqrt{F\left(\phi_{i j}^{* *}\right)}$.

In summary, we obtain the following numerical schemes of Eqs. (2.1a)-(2.1c) and then we solve these equations in order:

$$
\begin{aligned}
& \rho^{n} \frac{\mathbf{u}^{n+1}-\mathbf{u}^{n}}{\Delta t} \\
& =-\rho^{n}\left(\mathbf{u} \cdot \nabla_{d} \mathbf{u}\right)^{n}-\nabla_{d} p^{n+1}+\frac{1}{R e} \nabla \cdot\left[\eta\left(\phi^{n}\right)\left(\nabla_{d} \mathbf{u}^{n}+\nabla_{d}\left(\mathbf{u}^{n}\right)^{T}\right)\right] \\
& +2 \mathbf{S F}^{n}+\frac{\rho^{n}}{F r} \mathbf{g} \\
& \nabla_{d} \cdot \mathbf{u}^{n+1}=0 \text {, } \\
& \frac{\phi^{*}-\phi^{n}}{\Delta t}=\frac{\epsilon^{2}}{P e} \Delta_{d} \phi^{*}-\nabla_{d} \cdot(\phi \mathbf{u})^{n}, \\
& \phi^{* *}=\frac{\phi^{*}}{\sqrt{e^{-\frac{2 \Delta t}{P e}}+\left(\phi^{*}\right)^{2}\left(1-e^{-\frac{2 \Delta t}{P e}}\right)}}, \\
& \frac{\phi^{n+1}-\phi^{* *}}{\Delta t}=\beta^{* *} \sqrt{F\left(\phi^{* *}\right)} \text {. }
\end{aligned}
$$

Here,

$$
\beta^{* *}=\frac{1}{\Delta t} \sum_{i=1}^{N_{x}} \sum_{j=1}^{N_{y}}\left(\phi_{i j}^{0}-\phi_{i j}^{* *}\right) / \sum_{i=1}^{N_{x}} \sum_{j=1}^{N_{y}} \sqrt{F\left(\phi_{i j}^{* *}\right)}
$$




\section{Numerical experiment}

Unless otherwise specified, we use the following initial conditions, computational domains and boundary conditions. We let the inner and out fluids are both fluid 1 . The conditions are a circular droplet and zero velocities on the computational domain $\Omega=(0,2) \times(0,4)$ :

$$
\begin{aligned}
& \phi(x, y, 0)=\tanh \left(\frac{0.5-\sqrt{(x-1)^{2}+(y-1)^{2}}}{\sqrt{2} \epsilon}\right), \\
& u(x, y, 0)=v(x, y, 0)=0 .
\end{aligned}
$$

On the top and bottom boundaries, we take the zero-Neumann boundary condition for the order parameter $\phi$ and chemical potential $\mu$. The no-slip conditions are used for $u$ and $v$. As for the pressure $p$, we take the inner product of both sides in Eq. (2.1a) with the unit normal vector $\mathbf{n}$ :

$$
\mathbf{n} \cdot \nabla p=\mathbf{n} \cdot\left(\frac{1}{R e} \nabla \cdot\left[\eta(\phi)\left(\nabla \mathbf{u}+\nabla \mathbf{u}^{T}\right)\right]+2 \mathbf{S F}(\phi)+\frac{\rho(\phi)}{F r} \mathbf{g}\right) .
$$

On the left and right boundaries, we impose the periodic boundary condition for all variables. Across interfacial transition layer $\phi$ varies from -0.9 to 0.9 over a length of about $2 \sqrt{2} \epsilon \tanh ^{-1}(0.9)$. Therefore, if we want this value to be about $h \mathrm{~m}$, then $\epsilon=\epsilon_{m}=h m /\left[2 \sqrt{2} \tanh ^{-1}(0.9)\right]$ [26]. In our model, the fluid 2 locates in the region where $-0.9<\phi<0$ and occupies $h \mathrm{~m} / 2$. We set the width of fluid 2 to be $\delta=4 h$ in the following numerical experiments, therefore $\epsilon=\epsilon_{8}$ is used. The schematic illustrations are shown in Figs. 4(a) and (b).

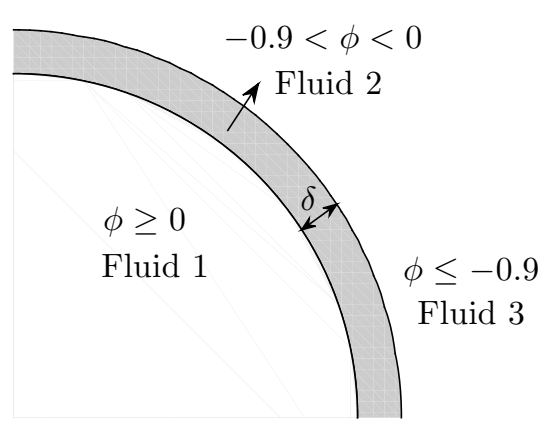

(a)

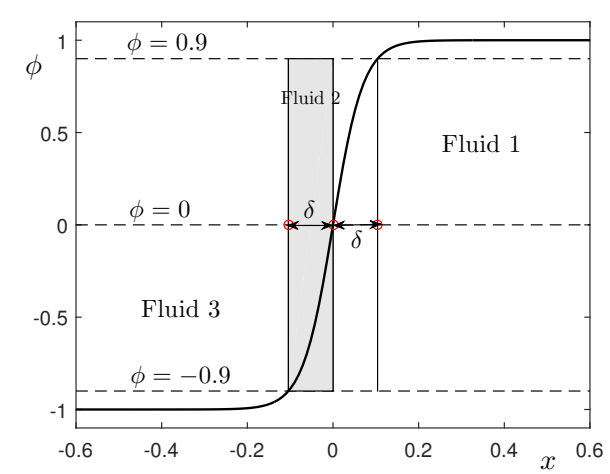

(b)

Figure 4: Schematic illustrations of (a) antibubble and (b) diffusive region, where the shadow region is filled with fluid 2 and $\delta$ represents the width of fluid 2 .

\subsection{Effect of Peclet number}

First, we investigate the effect of Peclet number by simulating the single bubble raising with $h=1 / 64, \Delta t=0.001, \epsilon=\epsilon_{8}, R e=30, W e=30, F r=1, \rho_{1}: \rho_{3}=1: 1$, 


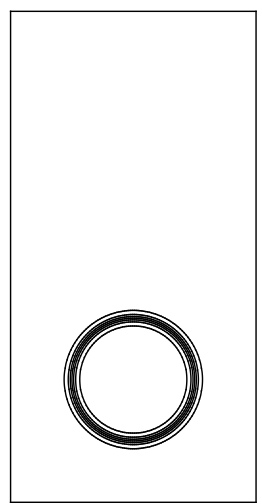

(a)

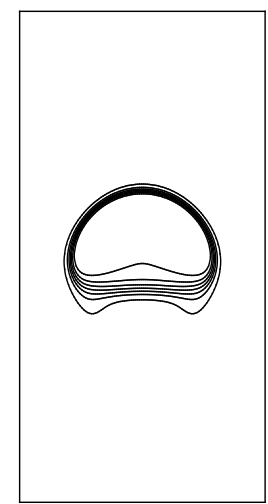

(b)

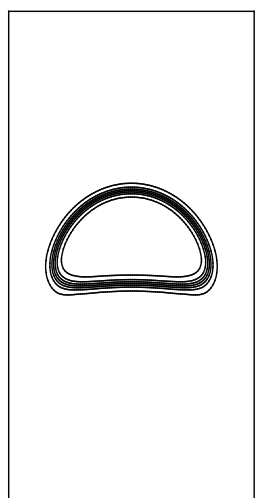

(c)

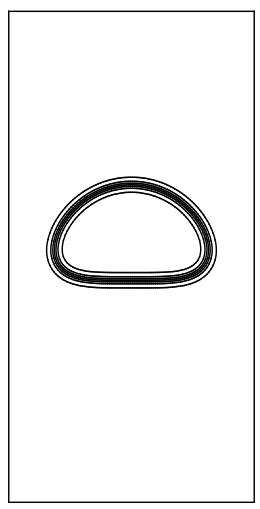

(d)

Figure 5: The effect of Peclet number. (a) Initial state, (b) $P e=0.1 / \epsilon$ at $t=5$, (c) $P e=0.01 / \epsilon$ at $t=5$ and (d) $P e=0.001 / \epsilon$ at $t=5$. The contour levels are $-0.9,-0.6,-0.3,0,0.3,0.6,0.9$.

$\rho_{1}: \rho_{2}=3: 1, \eta_{1}: \eta_{3}=1: 1$ and $\eta 1: \eta_{2}=3: 1$. Various Peclet numbers, $P e=0.1 / \epsilon, 0.01 / \epsilon$ and $0.001 / \epsilon$, are used. Fig. 5(a) shows the interfacial contours $(\phi=$ $-0.9,-0.6,-0.3,0,0.3,0.6,0.9)$ of the initial state. Figs. 5(b)-(d) show the interfacial contours for $P e=0.1 / \epsilon, 0.01 / \epsilon$ and $0.001 / \epsilon$ at $t=5$, respectively. We can find that a larger Peclet number causes a nonuniform distribution of contour lines, while a smaller Peclet number causes a dominant effect of the conservative Allen-Cahn equation. In our model, the middle fluid film is defined in the diffusive region where $\phi$ varies form -0.9 to 0 , therefore, the uniform distribution of contours is important. Unless otherwise stated, we will use $P e=0.01 / \epsilon$ in the following tests.

\subsection{The convergence of polygonal area with different mesh sizes}

We investigate the convergence of polygonal area of antibubble for different mesh sizes: $32 \times 64,64 \times 128$ and $128 \times 256 . \Delta t=0.001, \epsilon=0.03, R e=30, W e=30, F r=1$, $\rho_{1}: \rho_{3}=1: 1, \rho_{1}: \rho_{2}=3: 1, \eta_{1}: \eta_{3}=1: 1$ and $\eta_{1}: \eta_{2}=3: 1$ are used in this test. We define the polygonal area of antibubble as

$$
A=\left|\sum_{s=1}^{M}\left(X_{s} Y_{s+1}-X_{s+1} Y_{s}\right) / 2\right|
$$

(see Fig. 6), where $M$ is the total number of polygonal points on $\phi=-0.9$ level set. The relative error of polygonal area is defined as $\left|A^{n} / A^{0}-1\right|$. Fig. 7 shows the temporal evolutions of relative error of polygonal area for different mesh sizes. We can find that the relative error of polygonal area is close to 0 with $128 \times 256$. The polygonal area converges with refined mesh sizes. 


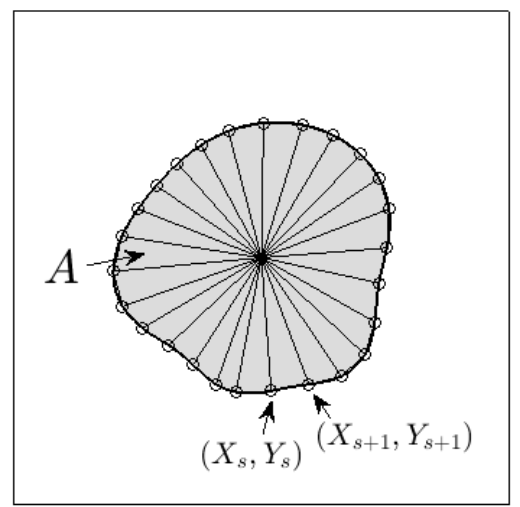

Figure 6: Schematic illustration of polygonal area.

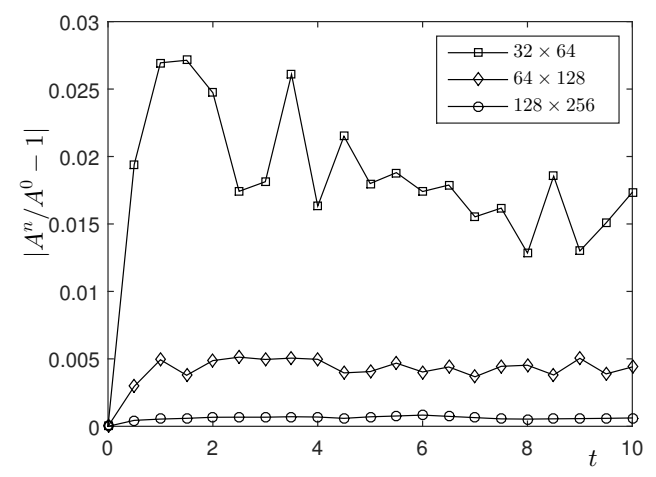

Figure 7: Temporal evolutions of the relative errors of polygonal area of antibubble for different mesh sizes.

\subsection{Advantage of the proposed model}

In order to verify the advantage of the proposed model, we compare the evolutions of antibubble raising using the proposed model and the conventional CAC model. In the convectional model, the initial condition is set to be an annular of fluid 2 , the rest region is filled by fluid 1 . The density and viscosity are defined as $\rho(\phi)=\rho_{1}(1-\phi) / 2+$ $\rho_{2}(1+\phi) / 2, \eta(\phi)=\eta_{1}(1-\phi) / 2+\eta_{2}(1+\phi) / 2$. In this test, we use the mesh size $128 \times 256, \Delta t=0.001, \epsilon=\epsilon_{8}, P e=0.01 / \epsilon, R e=30, W e=30$, and $F r=1$. The density and viscosity ratios for fluid 1 and fluid 2 are $3: 1$. Figs. $8(a)$ and (b) show the temporal evolution for the conventional model and the proposed model. In the conventional model, the sufficiently fine mesh size is needed to preserve the annular region; otherwise, the breakup of annular will occur and the nonphysical evolution can be found as shown in Fig. 8(a). However, the proposed model uses the diffusive region, $-0.9<\phi<0$, to represent the annular fluid, therefore, this approach naturally prevents the nonphysical breakup and the stable temporal evolution can be found in Fig. 8(b). 
(a)
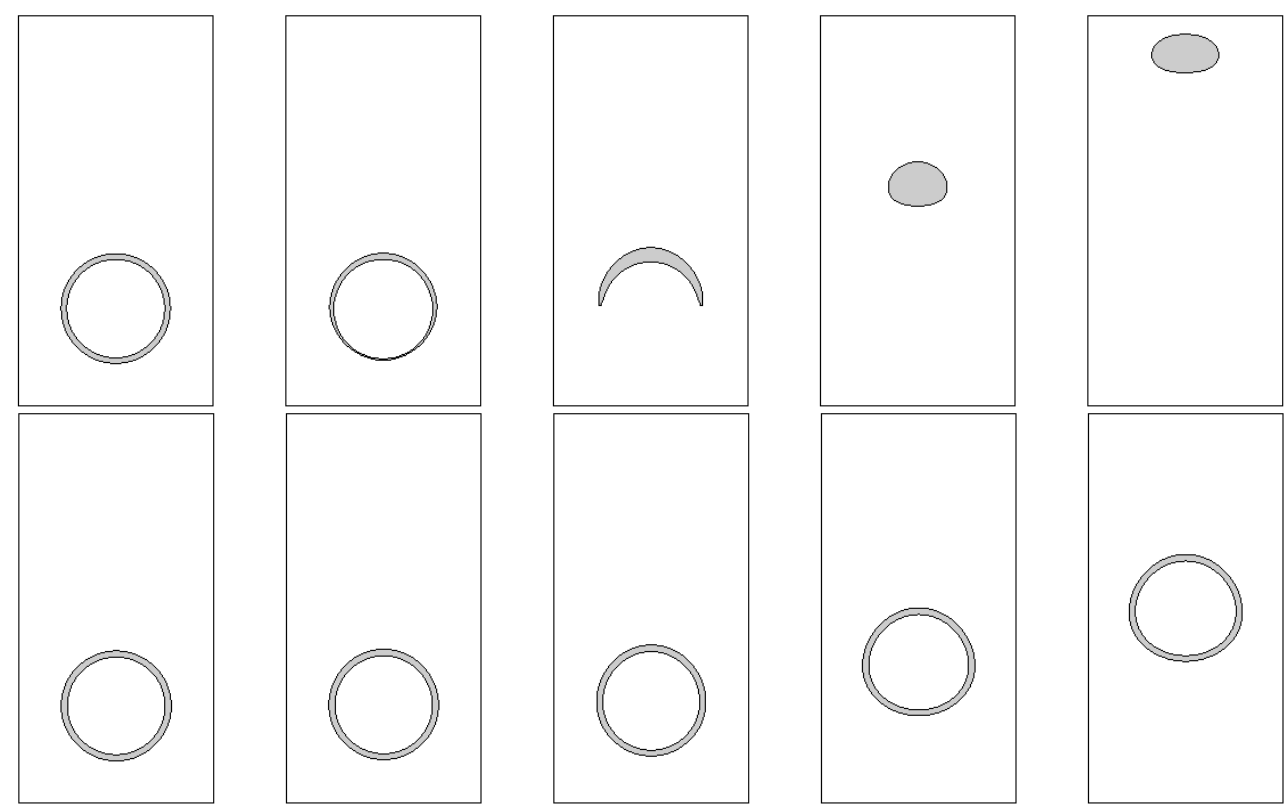

Figure 8: Temporal evolutions of antibubble by using (a) the conventional model and (b) the proposed model. The times from left to right are $t=0,1,2,7,13$.

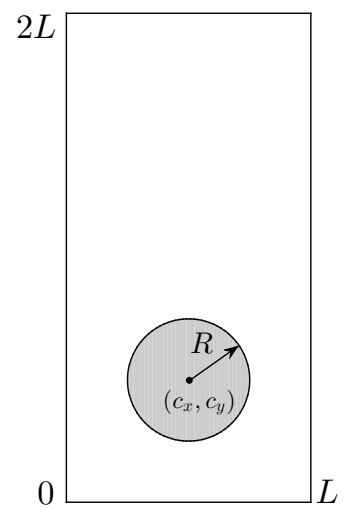

(a)

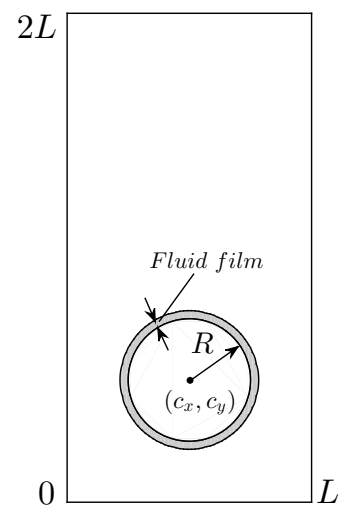

(b)

Figure 9: Schematic illustrations of (a) bubble and (b) antibubble rising in liquid.

\subsection{Comparison between the raising phenomenons of bubble and antibubble}

In this part, we compare the different dynamics of bubble and antibubble raising. The schematic illustration of the bubble and antibubble is depicted in Fig. 9. A bubble with radius $R=0.5$ is placed at $(1,1)$ in a rectangular domain $\Omega=(0,2) \times(0,4)$. For the bubble, $\rho_{3}$ vanishes, so we set $\rho_{1}: \rho_{2}=3: 1, \eta_{1}: \eta_{2}=3: 1$. For the antibubble, we 


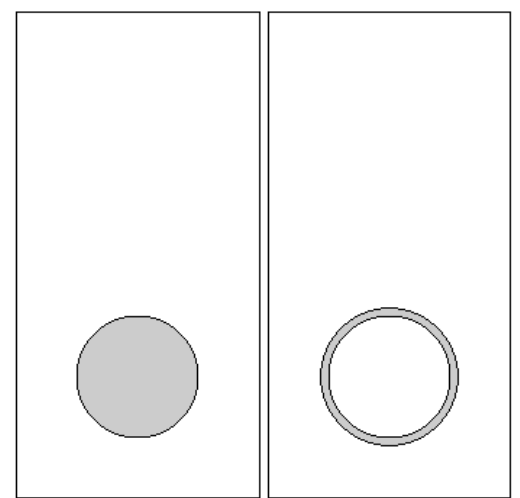

Bubble

(a)

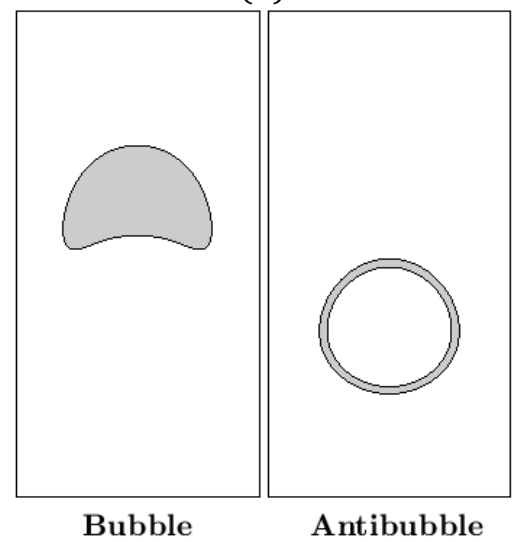

(c)

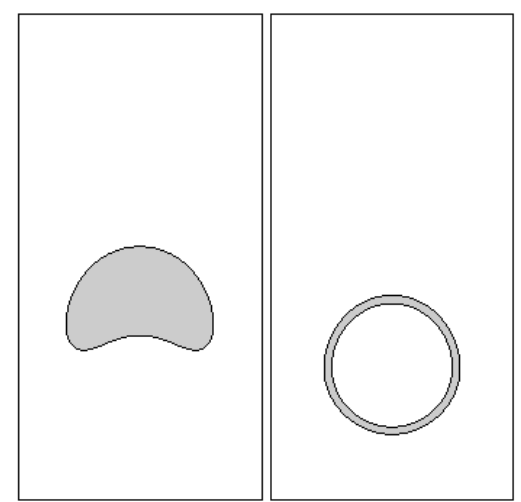

Antibubble

(b)

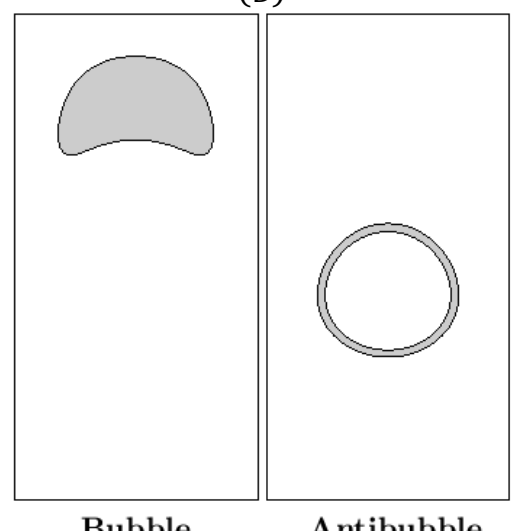

(d)

Figure 10: Temporal evolutions of bubble and antibubble. The times from (a) to (d) are at $t=0,3,6.5,10$.

use $\rho_{1}: \rho_{3}=1: 1, \rho_{1}: \rho_{2}=3: 1, \eta_{1}: \eta_{3}=1: 1$, and $\eta_{1}: \eta_{2}=3: 1$. The mesh size is $128 \times 256$. Other numerical parameters are $h=1 / 64, \Delta t=0.001, R e=30, W e=30$, $F r=1, \epsilon=\epsilon_{8}$, and $P e=0.01 / \epsilon$. Fig. 10 shows the temporal evolutions of bubble and antibubble. The bubble is defined in the gray region and the other fluid is defined in the white region. As we can see, bubble raises faster with larger deformation than the antibubble at the same times.

\subsection{Effect of density ratio}

In order to investigate the effect of density ratio between fluid 1 and fluid 2 . We choose three different density ratios: $\rho_{1}: \rho_{2}: \rho_{3}=2: 1: 2,3: 1: 3$ and $4: 1: 4$. The viscosity ratio for fluid 1 and fluid 2 is $1: 1$. We use the mesh size $128 \times 256$, $\Delta t=0.0002, \epsilon=\epsilon_{8}, P e=0.01 / \epsilon, R e=30, W e=30$, and $F r=1$. The initial condition 


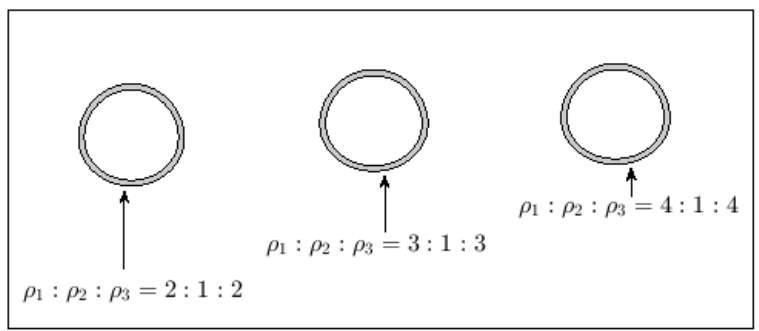

Figure 11: The snapshots of antibubble with different density ratios at $t=10$.

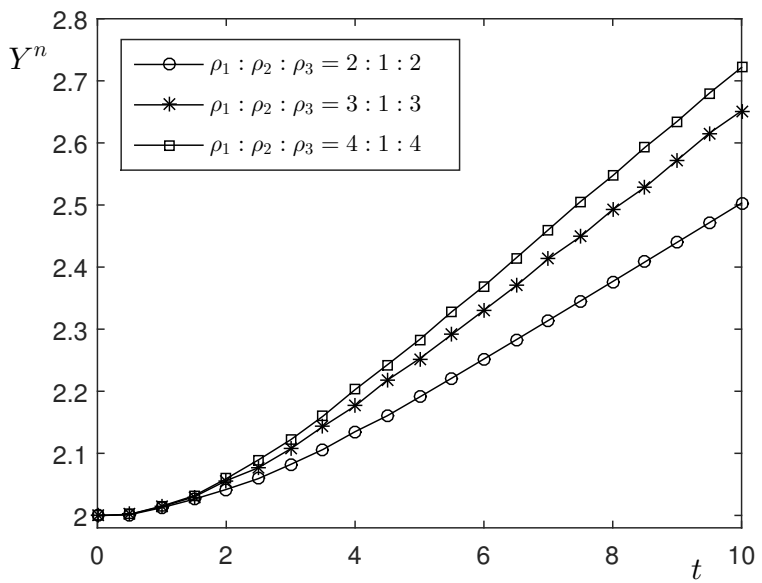

Figure 12: Temporal evolutions of the $y$-coordinate of the center of mass $\left(Y^{n}\right)$ in antibubble. $\rho_{1}: \rho_{2}: \rho_{3}=$ $2: 1: 2$ (line with circle markers), $\rho_{1}: \rho_{2}: \rho_{3}=3: 1: 3$ (line with star markers), $\rho_{1}: \rho_{2}: \rho_{3}=4: 1: 4$ (line with square markers).

is taken as

$$
\phi(x, y, 0)=\tanh \left(\frac{0.5-\sqrt{(x-1)^{2}+(y-2)^{2}}}{\sqrt{2} \epsilon}\right)
$$

on $\Omega=(0,2) \times(0,4)$. The computation runs until $t=10$. Fig. 11 shows the snapshots of antibubble for different density ratios at $t=10$. The temporal evolutions of the y-coordinate of the center of mass $\left(Y^{n}\right)$ of antibubble are shown in Fig. 12. If the difference of density ratio is larger, then the buoyancy effect is more pronounced and the antibubble with a larger density ratio is raising faster.

\subsection{Effect of viscosity ratio}

We investigate the effect of viscosity ratio. The inner fluid and outer fluid are set to be the same as fluid 1, i.e., $\eta_{1}=\eta_{3}$. The viscosity of fluid 2 is fixed as $\eta_{2}=0.1$. Five different viscosities for fluid 1 are used: $\eta_{1}=0.1,0.2,0.3,0.4,0.5$. The density ratio is set to be $3: 1: 3$ for all tests. We use the mesh size $128 \times 256, \Delta t=0.0002, \epsilon=\epsilon_{8}$, 


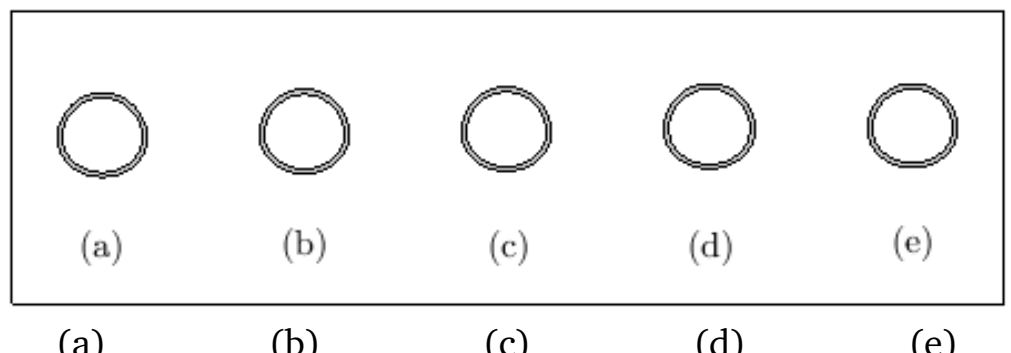

(a)

(b)

(c)

(d)

(e)

Figure 13: The snapshots of antibubble for different viscosity ratios at $t=10$. (a)-(e) represent the viscosity ratios: $\eta_{1}: \eta_{2}: \eta_{3}=1: 1: 1,2: 1: 2,3: 1: 3,4: 1: 4,5: 1: 5$, respectively.

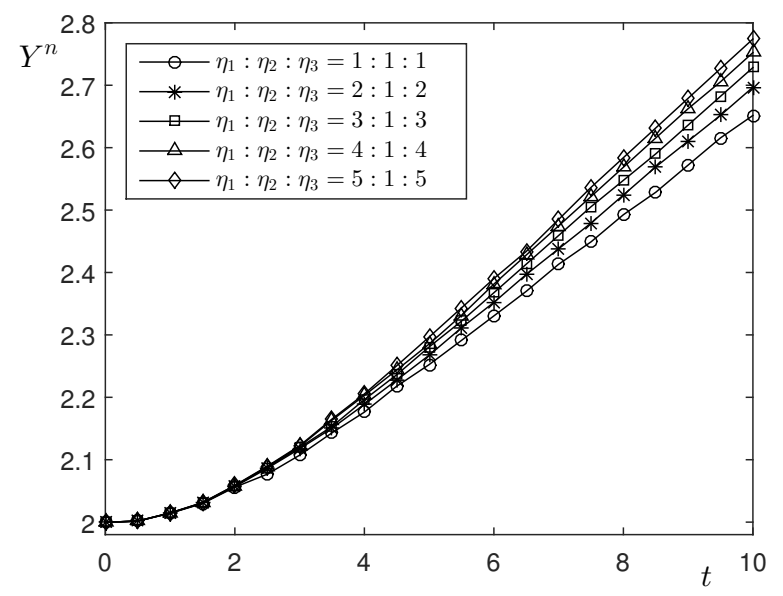

Figure 14: Temporal evolutions of the $y$-coordinate of the center of mass in antibubble for different viscosity ratios.

$P e=0.01 / \epsilon, R e=30, W e=30$, and $F r=1$. The initial condition is taken to be the same as in Eq. (4.1). The computation runs until $t=10$. The snapshots of antibubble for different viscosity ratios at $t=10$ are shown in Fig. 13. Fig. 14 shows the temporal evolutions of the y-coordinate of the center of mass in antibubble for different viscosity ratios. Although the difference is not significant, a larger viscosity ratio makes the antibubble rise faster. The existence of fluid 2 causes the raising of antibubble, a larger viscosity ratios leads to a smaller viscosity effect of fluid 2 , therefore, the raising with a larger viscosity ratio is faster than that with a smaller viscosity ratio.

\subsection{Effect of Weber number}

Next, we investigate the effect of Weber number $(W e)$ on the antibubble raising. The Weber number is an important parameter related to the surface tension, a larger value of Weber number reflects a smaller effect of surface tension while a smaller value of Weber number causes a larger surface tension. In this test, we use two different 
(a)
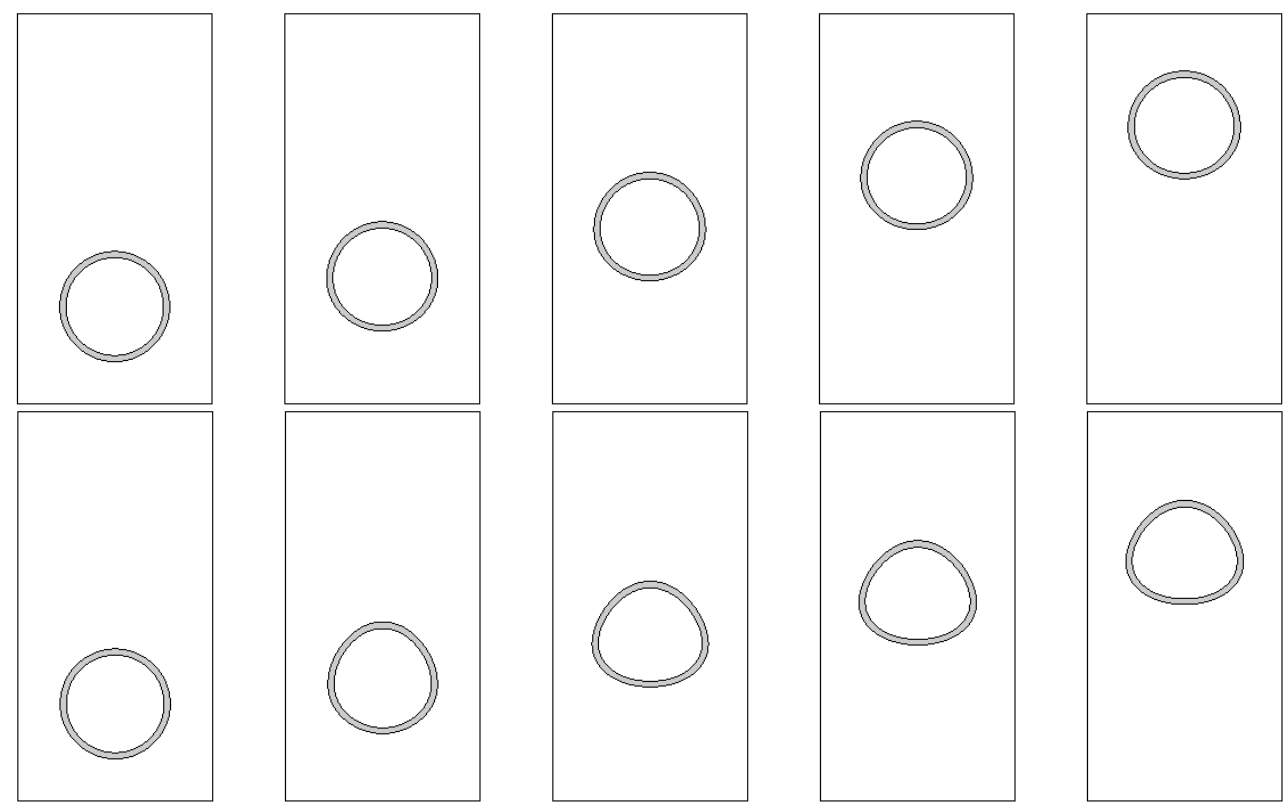

Figure 15: Temporal evolutions of antibubble for (a) $W e=10$ and (b) $W e=100$. The times from left to right are at $t=0,5,10,15,20$.

Weber numbers: $W e=10$ and $W e=100$. The other parameters are taken the same values as in Section 4.4. Figs. 15(a) and (b) show the temporal evolutions of antibubble for $W e=10$ and $W e=100$, respectively. As we can see, when $W e=10$, the surface tension is dominant, the deformation of antibubble is small. However, when $W e=100$, the deformation is large during the evolution. Although the deformation is large, there is no nonphysical breakup of fluid 2 in the whole process.

\subsection{Effect of the density of inner fluid}

While the densities of inner fluid and outer fluid are equal, the antibubble will raises with time since the buoyancy of middle fluid film. Here, we investigate the effect of the density of inner fluid by using three different density ratios: $\rho_{1}: \rho_{2}: \rho_{3}=3: 1$ : $3,4: 1: 3$ and $5: 1: 3$, where $\rho_{1}, \rho_{2}$ and $\rho_{3}$ represent the densities of inner, middle and outer fluid, respectively. The viscosity ratio for inner, middle and outer fluid is $3: 1: 3$, respectively. The other parameters are taken to be the same as in Section 4.4. The initial condition is

$$
\phi(x, y, 0)=\tanh \left(\frac{0.5-\sqrt{(x-1)^{2}+(y-2)^{2}}}{\sqrt{2} \epsilon}\right) .
$$

The temporal evolutions of antibubble with different inner densities are shown in Fig. 16. In addition, we define the temporal deformation rate: $D^{n}=(B / H)^{n}$, where 
(a)

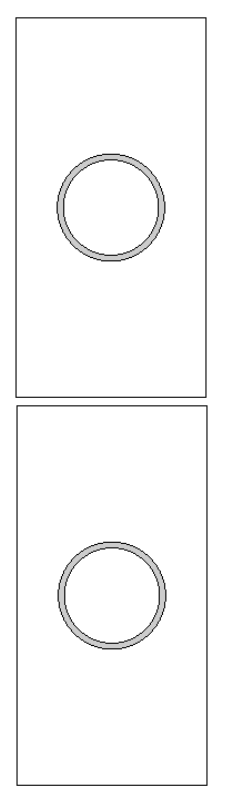

(b)

(c)

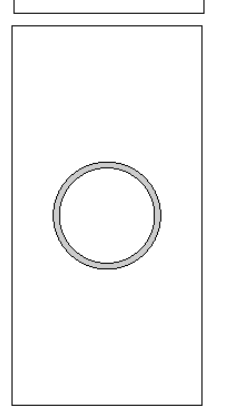

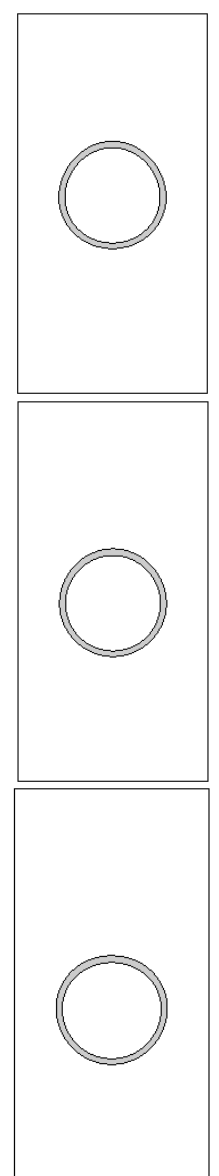
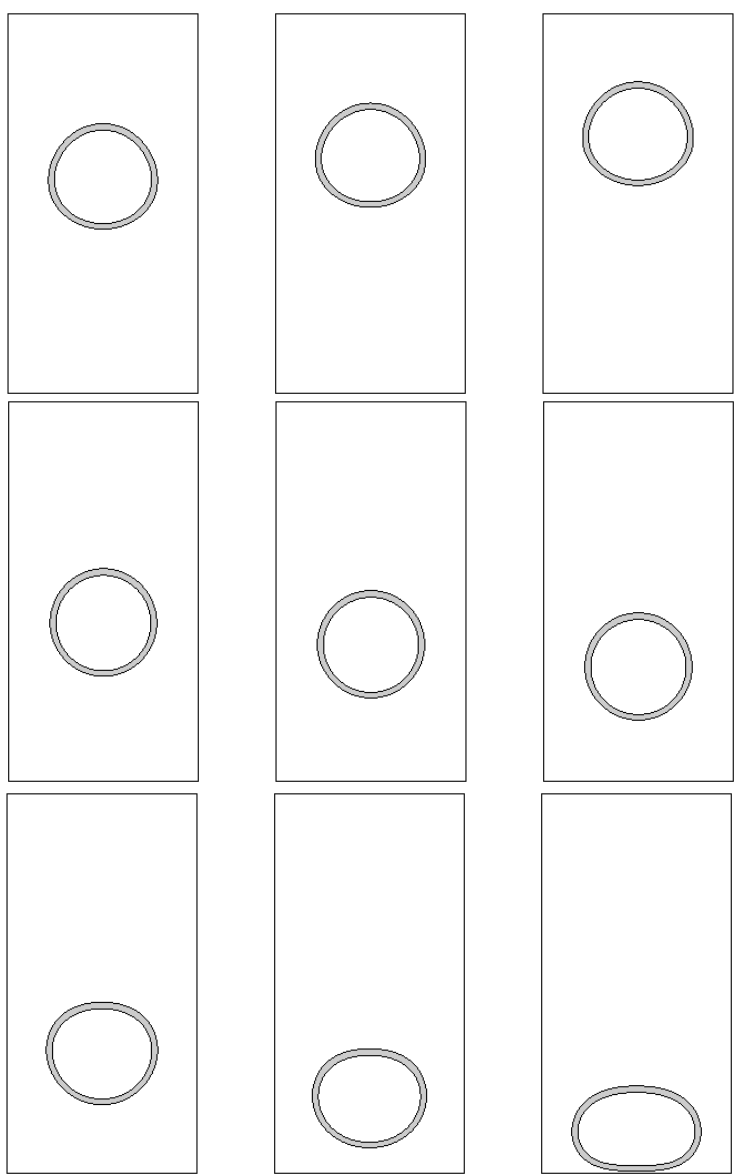

Figure 16: Temporal evolutions of antibubble with different inner densities. (a) $\rho_{1}: \rho_{2}: \rho_{3}=3: 1: 3$, (b) $\rho_{1}: \rho_{2}: \rho_{3}=4: 1: 3$, (c) $\rho_{1}: \rho_{2}: \rho_{3}=5: 1: 3$. The times from left to right are at $t=0,5,10,15,20$.

$B$ is the distance between $x_{\max }$ and $x_{\min }$ and $H$ is the distance between $y_{\max }$ and $y_{\min }$. Here, $x_{\max }$ and $x_{\min }$ are the maximum and minimum of $x$-coordinate on the antibubble interface, $y_{\max }$ and $y_{\min }$ are the maximum and minimum of $y$-coordinate on the antibubble interface (see Fig. 17(a)). Fig. 17(b) illustrates the temporal evolutions of deformation rate with three different inner densities. When the inner density is large than the outer density, buoyancy does not balance the gravity and the antibubble starts to falling with time. We can observe that the deformation rate is not significant when the inner density is 4 . With the increase of inner density, the falling speed becomes fast and the deformation rate becomes larger.

\subsection{Temporal evolution of antibubble with initial rotation velocity field}

Finally, we investigate the dynamics of antibubble with initial rotation velocity field. The numerical parameters are $h=1 / 64, \Delta t=0.001, R e=20, W e=1, P e=0.01 \epsilon$, 


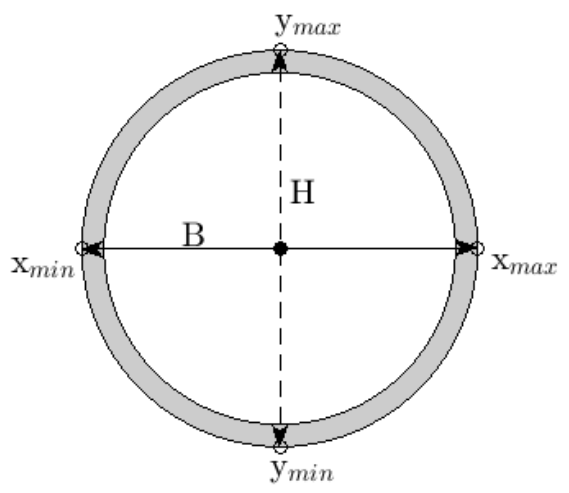

(a)

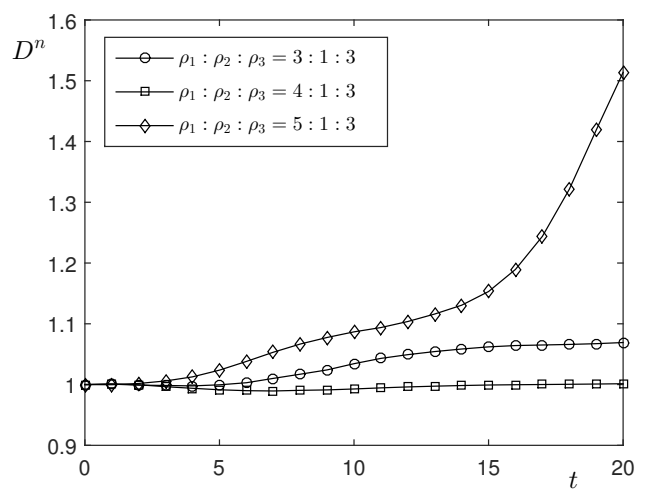

(b)

Figure 17: (a) Schematic diagram. (b) Temporal evolutions of deformation rate with different inner densities.

$\epsilon=\epsilon_{8}$, and $F r=1$. The initial conditions are taken to be

$$
\begin{aligned}
& \phi(x, y, 0)=\tanh \left(\frac{0.25-\sqrt{(x-1)^{2}+(y-1.4)^{2}}}{\sqrt{2} \epsilon}\right) \\
& u(x, y, 0)=-\sin ^{2}(0.5 \pi x) \sin (\pi y) \\
& v(x, y, 0)=\sin (\pi x) \sin ^{2}(0.5 \pi y)
\end{aligned}
$$

on the domain $\Omega=(0,2) \times(0,2)$. No-slip boundary condition for velocities is imposed on all boundaries. The temporal evolution of antibubble is shown in Fig. 18. Because the existence of the initial velocity, the antibubble first rotates and moves downward along the velocity direction. With the effect of buoyancy, the downward velocity becomes weaker gradually and then the antibubble raises with time.

\section{Conclusions}

A new mathematical model for simulating the dynamics of antibubble was proposed in this work. The conservative Allen-Cahn equation was adopted in this model and the inner, middle and outer fluids are defined in different diffusive regions. The corresponding density and viscosity functions were presented to fit this new model. Numerical results demonstrated that the proposed model can simulate the antibubble without nonphysical breakup. In addition, the effects of various numerical parameters on the dynamics of antibubble were also investigated.

Acknowledgements The author (D. Jeong) was supported by the National Research Foundation of Korea (NRF) grant funded by the Korea government (MSIP) (NRF2017R1E1A1A03070953). The author (Y. B. Li) is supported by National Natural Science Foundation of China (Nos. 11601416, 11631012) and by the China Postdoctoral 


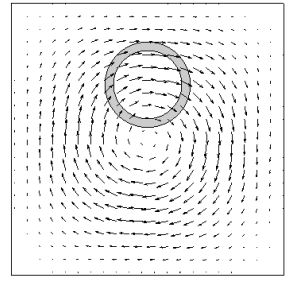

(a)

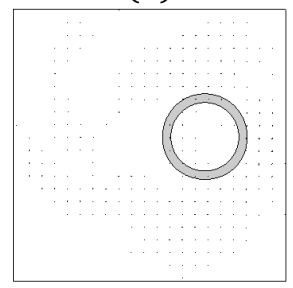

(e)

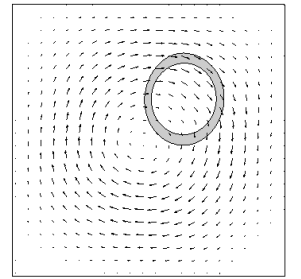

(b)

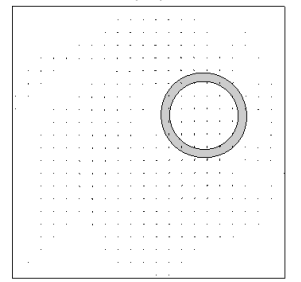

(f)

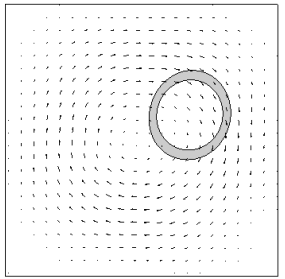

(c)

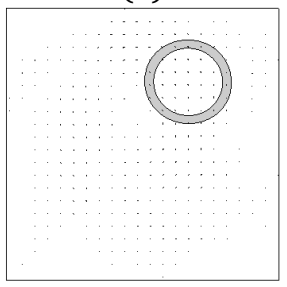

(g)

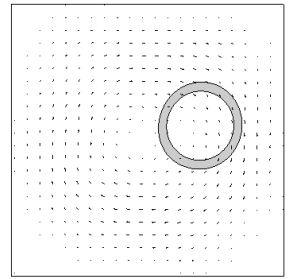

(d)

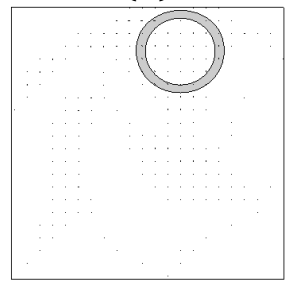

(h)

Figure 18: Temporal evolutions of antibubble with an initial rotation velocity field. The velocities are scaled by 0.1 for a better visualization. The times from $(\mathrm{a})$ to $(\mathrm{h})$ are at $t=0,1,2,3,5,9,14,20$.

Science Foundation (No. 2018M640968). The corresponding author (J. S. Kim) was supported by Basic Science Research Program through the National Research Foundation of Korea (NRF) funded by the Ministry of Education (NRF-2019R1A2C1003053).

\section{References}

[1] P. G. Kim And H. A. Stone, Dynamics of the formation of antibubbles, EPL, 83 (2008), 54001.

[2] C. L. Strong, Stories by C. L. Stone, Sci. Am., 230 (1974), 116.

[3] K. P. Galvin, S. J. Pratten, G. M. Evans and S. Biggs, Spontaneous formation of an "antidrop", Langmuir, 22 (2006), pp. 522-523.

[4] P. WeIss, The rise of antibubbles, Sci. News, 165 (2004), pp. 311-313.

[5] B. Scheid, J. Zawala AND S. Dorbolo, Gas dissolution in antibubble dynamics, Soft Matter, 10 (2014), 7096.

[6] J. E. SilPE AND D. W. MCGRAIL, Magnetic antibubbles: Formation and control of magnetic macroemulsions for fluid transport applications, J. Appl. Phys., 113 (2013), pp. 17B304.

[7] L. LAURIER, Schramm, Emulsions, Foams and Suspensions: Fundamentals and Applications, Strauss GmbH, Mörlenbach, (2005).

[8] E. Dluska, A. Markowska-Radomska, A. Metera and W. Tomaszewski, Drug-core double emulsions for co-release of active ingredients, Int. J. Chem. Eng. Appl., 7(6) (2016), pp. 428-432.

[9] B. Scheid, S. Dorbolo, L. R. ARriaga And E. Rio, Antibubble dynamics: the drainage of an air film with viscous interfaces, Phys. Rev. Lett., 109 (2012), 264502.

[10] D. N. Sob'Yanin, Theory of the antibubble collapse, Phys. Rev. Lett., 114 (2015), 104501.

[11] J. Zou, C. Ji, B. G. YuAn, X. D. RuAn AND X. Fu, Collapse of an antibubble, Phys. Rev. E, 87 (2013), 061002(R). 
[12] L. BAI, W. XU, P. WU, W. LIN, C. LI AND D. XU, Formation of antibubbles and multilayer antibubbles, Colloid Surface A, 509 (2016), pp. 334-340.

[13] J. KIm, Modeling and Simulation of Multi-Component, Multi-Phase Fluid Flows: Twin Cities, University of Minnesota.

[14] T. Mitchell, C. LEONARDi AND A. FAKHARI, Development of a three-dimensional phasefield lattice Boltzmann method for the study of immiscible fluids at high density ratios, Int. J. Multiphase Flow, 107 (2018), pp. 1-15.

[15] N. Talet, B. Mavric, G. Belsa, V. Hatic, S. Bajt and B. Sarler, Development of meshless phase field method for two-phase flow, Int. J. Multiphase Flow, 108 (2018), pp. 169-180.

[16] K. Park, M. Fernandino And C. A. Dorao, Thermal two-phase flow with a phase-field method, Int. J. Multiphase Flow, 100 (2018), pp. 77-85.

[17] J. YANG AND J. Kim, Phase-field simulation of Rayleigh instability on a fibre, Int. J. Multiphase Flow, 105 (2018), pp. 84-90.

[18] Z. YANG AND S. DONG, Multiphase flows of $N$ immiscible incompressible fluids: An outflow/open boundary condition and algorithm, J. Comput. Phys., 366 (2018), pp. 33-70.

[19] D. JEONG AND J. Kim, Conservative Allen-Cahn-Navier-Stokes system for incompressible two-phase fluid flows, Comput. Fluids, 156 (2017), pp. 239-246.

[20] J. KIM, A continuous surface tension force formulation for diffuse-interface models, J. Comput. Phys., 204(2) (2005), pp. 784-804.

[21] H. G. LEE, K. KIM AND J. KIM, On the long time simulation of the Rayleigh-Taylor instability, Int. J. Numer. Meth. Eng., 85(13) (2011), pp. 1633-1647.

[22] G. Vilanova, I. Colominas And H. Gomez, A mathematical model of tumour angiogenesis: growth, regression and regrowth, J. R. Soc. Interface, 14(126) (2017), 20160918.

[23] A. J. CHORIN, A numerical method for solving incompressible viscous flow problems, J. Comput. Phys., 135(2) (1997), pp. 118-125.

[24] J. Kim, S. LEE AND Y. CHOI, A conservative Allen-Cahn equation with a space-time dependent Lagrange multiplier, Int. J. Eng. Sci., 84 (2014), pp. 11-17.

[25] U. Trottenberg, C. Oosterlee And A. Schüller, Multigrid, Academic Press, New York, (2001).

[26] J. W. ChoI, H. G. LeE AND J. KIM, An unconditionally gradient stable numerical method for solving the Allen-Cahn equation, Phys. A, 388 (2009), pp. 1791-1803. 